\title{
ALMOST DIRECT SUMMANDS
}

\author{
BHARGAV BHATT
}

\begin{abstract}
We prove new cases of the direct summand conjecture using fundamental theorems in $p$-adic Hodge theory due to Faltings. The cases tackled include the ones when the ramification locus lies entirely in characteristic $p$.
\end{abstract}

\section{$\S 1$. Introduction}

The direct summand conjecture of Hochster asserts that any modulefinite extension $R \rightarrow S$ of commutative rings with $R$ regular is a direct summand as an $R$-module map. This conjecture is known when $R$ contains a field (see [Ho1]) or if $\operatorname{dim}(R) \leq 3$ (see $[\mathrm{H}]$ ). The general mixed characteristic case remains wide open and is a fundamental open problem in commutative algebra; we refer the interested reader to [Ho3] for a discussion of various other conjectures implied by the direct summand conjecture.

Our goal in this article is to explain how Faltings's theory of almost étale extensions in $p$-adic Hodge theory can be used to prove new cases of the direct summand conjecture. Most notably, this method applies to maps whose ramification locus has simple normal crossings after inverting $p$; that is, we impose no requirements on the characteristic $p$ ramification. In particular, there is no constraint on the dimension.

\section{The statement}

Fix a prime number $p$, and let $V$ be a complete $p$-adic discrete valuation ring whose residue field $k$ satisfies $\left[k: k^{p}\right]<\infty$; for example, we could take $V$ to be a finite extension of $\mathbf{Z}_{p}$. We will prove the following.

TheOREM 1.1. Let $R$ be a smooth $V$-algebra, and let $f: R \rightarrow S$ be the normalization of $R$ in a finite extension of its fraction field. Assume that there exists an étale map $V\left[T_{1}, \ldots, T_{d}\right] \rightarrow R$ such that $f \otimes_{R} R\left[\left(1 / p \cdot T_{1}, \ldots, T_{d}\right)\right]$ is unramified. Then $f: R \rightarrow S$ is a direct summand as an $R$-module map.

Received February 18, 2013. Accepted May 14, 2013.

First published online March 25, 2014.

2010 Mathematics Subject Classification. Primary 13H05; Secondary 13D22, 14B05. 
We will prove Theorem 1.1 using a deep purity result of Faltings conceived in $p$-adic Hodge theory. To the best of our knowledge, Theorem 1.1, or even the special case where $f[(1 / p)]$ is unramified, is inaccessible to other methods used to study the direct summand conjecture. It is also worth noting that the general case of the direct summand conjecture can be reduced to base rings $R$ which are localizations of étale $V\left[T_{1}, \ldots, T_{d}\right]$-algebras with $k=\bar{k}$ (by [Ho2, Remark 6.5]), so the only serious assumption in Theorem 1.1 is the one on ramification. Finally, note that the almost ring theory approach to the direct summand conjecture adopted in this paper was also explored in $[R]$.

This article is organized as follows. In Section 2, we review the basics of almost ring theory and recall Faltings's almost purity result, the main ingredient of our proof of Theorem 1.1. We then prove the theorem in Section 3.

\section{$\S 2$. Review of almost ring theory}

Our proof uses almost ring theory as discovered by Tate $[\mathrm{T}]$ and developed by Faltings in [F1], [F2] with $p$-adic Hodge-theoretic applications in mind. The book [GR1] provides a systematic treatment of almost ring theory, and [O] provides a detailed and comprehensible presentation of the arithmetic applications of Faltings's ideas. We review below the aspects of this theory most relevant to the proof of Theorem 1.1, deferring to the other sources for proofs.

\subsection{Almost mathematics}

Let $\bar{V}$ denote a valuation ring whose value group $\Lambda$ is dense in $\mathbf{Q}$, and let $\mathfrak{m} \subset \bar{V}$ be the maximal ideal. Note that $\mathfrak{m}$ is necessarily not finitely generated. For each nonnegative $\alpha \in \Lambda$, let $\mathfrak{m}_{\alpha}$ be the (necessarily principal) ideal of elements of valuation at least $\alpha$, and let $\pi \in \bar{V}$ denote an element of valuation 1 .

EXAMPLE 2.1. Let $K$ denote the fraction field of a complete $p$-adic discrete valuation ring $V$, and let $\bar{V}$ denote the integral closure of $V$ in a fixed algebraic closure $\bar{K}$ of $K$. Then $\bar{V}$ is a valuation ring whose value group is $\mathbf{Q}$, and consequently, almost ring theory applies; this will be the only example relevant to us.

The maximal ideal $\mathfrak{m}$ can be thought of as the ideal of elements with positive valuation. As the value group is dense, it follows that $\mathfrak{m}^{2}=\mathfrak{m}$. This 
observation implies that the category $\Sigma$ of $\mathfrak{m}$-torsion $\bar{V}$-modules is a Serre subcategory of the abelian category $\operatorname{Mod}(\bar{V})$ of $\bar{V}$-modules. We refer to modules in $\Sigma$ as almost zero modules. By general nonsense, we may form the quotient abelian category

$$
\operatorname{Mod}(\bar{V})^{a}:=\operatorname{Mod}(\bar{V}) / \Sigma
$$

of almost $\bar{V}$-modules. We denote the localization functor by $M \mapsto M^{a}$. With this notation, we have the following description of maps in $\operatorname{Mod}(\bar{V})^{a}$ (see [GR1, Section 2.2.4]):

$$
\operatorname{Hom}_{\operatorname{Mod}(\bar{V})^{a}}\left(M^{a}, N^{a}\right)=\operatorname{Hom}_{\operatorname{Mod}(\bar{V})}\left(\mathfrak{m} \otimes_{\bar{V}} M, N\right) .
$$

As $\Sigma \subset \operatorname{Mod}(\bar{V})^{a}$ is closed under tensor products, the quotient $\operatorname{Mod}(\bar{V})^{a}$ inherits the structure of a symmetric $\otimes$-category with the quotient map $\operatorname{Mod}(\bar{V}) \rightarrow \operatorname{Mod}(\bar{V})^{a}$ being a symmetric $\otimes$-functor. This formalism allows one to systematically define almost analogues of standard notions of ring theory and, indeed, develop almost algebraic geometry. Informally, we may think of almost algebraic geometry as the study of algebraic geometry over $\bar{V}$ where all the results hold up to $\mathfrak{m}$-torsion. (For an example of how this program is carried out at the appropriate level of generality, see [GR1].) We will adopt the more pragmatic stance of explaining the notions we need to precisely state Faltings's almost purity theorem. We start with the following set of definitions, borrowed from [O, Section 2], which allow us to define the fundamental notion of an almost étale morphism.

Definition 2.2. Let $A$ be a $\bar{V}$-algebra, and let $M$ be an $A$-module. We say that

(1) $M$ is almost projective if $\operatorname{Ext}_{A}^{i}(M, N)$ is almost zero for all $A$-modules $N$ and if $i>0$;

(2) $M$ is almost flat if $\operatorname{Tor}_{i}^{A}(M, N)$ is almost zero for all $A$-modules $N$ and if $i>0$;

(3) $M$ is almost faithfully flat if it is almost flat and if, for any $A$-modules $N_{1}$ and $N_{2}$, the natural map

$$
\operatorname{Hom}_{R}\left(N_{1}, N_{2}\right) \rightarrow \operatorname{Hom}_{R}\left(N_{1} \otimes M, N_{2} \otimes M\right)
$$

has an almost zero kernel; 
(4) $M$ is almost finitely generated if for every strictly positive $\alpha \in \Lambda$ there exists a finitely generated $A$-module $N_{\alpha}$ and a $\pi^{\alpha}$-isomorphism $N_{\alpha} \simeq$ $M$; that is, there are maps $\phi_{\alpha}: N_{\alpha} \rightarrow M$ and $\psi_{\alpha}: M \rightarrow N_{\alpha}$ such that $\phi_{\alpha} \circ \psi_{\alpha}=\pi^{\alpha} \circ$ id and $\psi_{\alpha} \circ \phi_{\alpha}=\pi^{\alpha} \circ$ id.

REMARK 2.3. The properties defined in Definition 2.2 are all invariant under almost isomorphisms and, consequently, depend only on the almost isomorphism class $M^{a} \in \operatorname{Mod}(\bar{V})^{a}$. This is clear for flatness and projectivity by the exactness of the localization functor $\operatorname{Mod}(\bar{V}) \rightarrow \operatorname{Mod}(\bar{V})^{a}$. The issue of finite generation is more delicate, and we refer the interested reader to [GR1, Section 2.3] for a detailed discussion. We simply point out here that $\mathfrak{m} \subset \bar{V}$ is almost finitely generated with the definition given above.

WARning 2.4. As all the properties defined in Definition 2.2 are invariant under almost isomorphisms, it is tempting to define notions such as almost projectivity purely in terms of the internal homological algebra of the abelian category $\operatorname{Mod}(\bar{V})^{a}$ - that is, in terms of the internal Ext functors. However, this approach suffers from two defects. First, as the category $\operatorname{Mod}(\bar{V})^{a}$ lacks enough projectives (the generating object $\bar{V}^{a}$ is not projective), one is forced to resort to a Yoneda definition of the Ext groups, which is typically harder to work with. More serious, as the Yoneda definition pays no attention to the $\otimes$-structure, the resulting theory does not interact well with the $\otimes$ structure.

Once we have access to a good theory of flatness and finite generation, one can copy the standard notions in algebraic geometry to arrive at the fundamental notion of an almost étale morphism.

Definition 2.5. A morphism $A \rightarrow B$ of $\bar{V}$-algebras is called an almost étale covering if

(1) $B$ is almost finitely generated, almost faithfully flat, and almost projective as an $A$-module;

(2) $B$ is almost finitely generated and almost projective as a $B \otimes_{A} B$ module.

EXAMPLE 2.6. For completeness, we discuss the first nontrivial example of an almost étale morphism, which was discovered by Tate in his study of $p$ divisible groups [T]; we follow Faltings's exposition from [F1, Theorem 1.2]. Let $V$ be a finite extension of $\mathbf{Z}_{p}$, and let $\bar{V}$ be the normalization of $V$ in a fixed algebraic closure of its fraction field. Fix a tower $V=V_{0} \subset V_{1} \subset \cdots \subset$ 
$V_{n} \subset \cdots$ of normal subextensions of $\bar{V}$ such that $\Omega_{V_{n+1} / V_{n}}^{1}$ has $V_{n+1} / p$ as a quotient for each $n$; such towers can be produced starting with a totally ramified $\mathbf{Z}_{p}$-extension of $V$, and an explicit example may be constructed starting with the tower of extensions obtained by adjoining $p$-power roots of 1 . We set $V_{\infty}=\operatorname{colim}_{n} V_{n}$. Then $V_{\infty}$ can be shown to be a valuation ring whose value group is dense in $\mathbf{Q}$, and consequently, almost ring theory applies. The key observation (due to Tate, in different language) is that the natural map $V_{\infty} \rightarrow \bar{V}$ is almost étale (see [T, Section 3.2, Proposition 9]). A consequence of this fact is that many computations over $\bar{V}$ (such as those of certain Galois cohomology groups) can be reduced to computations over $V_{\infty}$, and the latter are typically much more tractable since $V_{\infty}$ can be chosen to have properties adapted to the problem at hand. The higherdimensional version of such arguments plays a key role in [F1].

Finally, we record a basic fact concerning the almost analogue of finite flat morphisms that is used in the proof of Theorem 1.1.

Lemma 2.7. Let $f: A \rightarrow B$ be an inclusion of $\bar{V}$-algebras. Assume that $f$ makes $B$ an almost projective and almost faithfully flat $A$-module. Then the cokernel coker $(f)$ is an almost projective A-module.

Proof. For any two $A$-modules $M$ and $N$, we have an identification of functors

$$
\mathrm{R} \operatorname{Hom}\left(M \otimes^{\mathrm{L}} N,-\right) \simeq \mathrm{R} \operatorname{Hom}(M, \mathrm{R} \operatorname{Hom}(N,-)) .
$$

A spectral sequence argument then shows that if $M$ and $N$ are almost projective, then so is $M \otimes^{\mathrm{L}} N$. If, in addition, one of $M$ or $N$ is also almost flat, then one has an almost isomorphism $M \otimes^{\mathrm{L}} N \simeq M \otimes N$. Hence, if both $M$ and $N$ are almost projective and one of them is almost flat, then $M \otimes N$ is almost projective. In particular, we see that $B \otimes_{A} B$ is almost projective. Now note that we have an exact sequence

$$
0 \rightarrow A \rightarrow B \rightarrow \operatorname{coker}(f) \rightarrow 0
$$

Tensoring this over $A$ with $B$ gives the new exact sequence

$$
0 \rightarrow B \rightarrow B \otimes_{A} B \rightarrow \operatorname{coker}(f) \otimes_{A} B \rightarrow 0 .
$$

The multiplication map on $B$ splits this exact sequence. Thus, $\operatorname{coker}(f) \otimes_{A}$ $B$ is a direct summand of the almost projective $A$-module $B \otimes_{A} B$ and, consequently, almost projective itself. By [GR1, Lemma 4.1.5], it follows that coker $(f)$ is also an almost projective $A$-module. 


\subsection{Faltings's purity theorem}

We now state the version of Faltings's almost purity theorem most relevant to Theorem 1.1: the case of good reduction with ramification supported along a simple normal crossings divisor. There exist more general statements than the one stated below - notably, the case of semistable reduction.

Let $V$ be a complete $p$-adic discrete valuation ring whose residue field $k$ satisfies $\left[k: k^{p}\right]<\infty$. Let $\bar{V}$ be the normalization of $V$ in a fixed algebraic closure of its fraction field. We will work with almost ring theory over $\bar{V}$. Let $R$ be a smooth $V$-algebra such that $R_{\bar{V}}:=R \otimes_{V} \bar{V}$ is a domain. Assume that we are given an étale morphism $V\left[T_{1}, \ldots, T_{d}\right] \rightarrow R$; such a chart always exists Zariski locally on $\operatorname{Spec}(R)$. Then we define a sequence of $R_{\bar{V}}$-algebras by

$$
R_{n}=\bar{V}\left[T_{i}^{1 / n !}\right] \otimes_{V\left[T_{i}\right]} R .
$$

One can check that the $\bar{V}$-algebras $R_{n}$ are finitely presented and smooth over $\bar{V}$ and, in particular, normal. Moreover, by construction, there are natural maps $R_{n} \rightarrow R_{m}$ for $n \leq m$, and we set $R_{\infty}=\operatorname{colim}_{n} R_{n}$ where the colimit is taken along the preceding maps. Note that $R_{\bar{V}} \rightarrow R_{n}$ is finite and faithfully flat, and consequently, $R_{\bar{V}} \rightarrow R_{\infty}$ is integral and faithfully flat. A calculation with differentials shows that the composite $R \rightarrow R_{\bar{V}} \rightarrow R_{\infty}$ is unramified away from the divisor $V\left(p \cdot T_{1}, \ldots, T_{d}\right)$. The purity theorem says that $R \rightarrow R_{\infty}$ is the maximal extension of $R$ with this last property, up to almost étale covers.

Theorem 2.8 ([F2, Section $2 \mathrm{~b}])$. Let $f: R \rightarrow S$ be the normalization of $R$ in a finite extension of its fraction field. Assume that the induced map $f \otimes_{R} R\left[\left(1 / p T_{1}, \ldots, T_{d}\right)\right]$ is étale. If $S_{n}$ denotes the normalization of $S \otimes_{R} R_{n}$ and if $S_{\infty}=\operatorname{colim}_{n} S_{n}$, then the induced map $R_{\infty} \rightarrow S_{\infty}$ is an almost étale covering.

REMARK 2.9. Theorem 2.8 can be thought of as a mixed characteristic analogue of Abhyankar's lemma without tameness restrictions. Recall that Abhyankar's lemma (see [Gro, Exposé XIII, Proposition 5.2]) asserts that, for any regular local ring $R$, the maximal extension of $R\left[T_{1}, \ldots, T_{d}\right]$ tamely ramified along the divisor associated to $T_{1} \cdots T_{d}$ may be obtained by adjoining all $n$-power roots of the $T_{i}$, where $n$ runs through integers invertible on $R$. The purity theorem does away with the tameness restrictions at the expense of describing the maximal extension ramified along a normal crossings divisor in mixed characteristic only up to almost étale covers. Most notably, it allows for one of the parameters $T_{i}$ to be replaced by $p$. 


\section{$\S 3$. Proof of Theorem 1.1}

Our goal in this section is to prove Theorem 1.1. Correspondingly, let $V$ be as in Theorem 1.1, and let $\bar{V}$ be the normalization of $V$ in a fixed algebraic closure of its fraction field. An essential idea informing the construction of almost ring theory is that the passage from algebraic geometry over $V$ to almost algebraic geometry over $\bar{V}$ is fairly faithful. We record one manifestation of this idea that will be useful to us.

Lemma 3.1. Let $R$ be a flat $V$-algebra essentially of finite type, and let $R_{\infty}$ be a faithfully flat $R_{\bar{V}}$-algebra. If $M$ is an $R$-module such that $M \otimes_{R} R_{\infty}$ is zero in $\operatorname{Mod}(\bar{V})^{a}$, then $M=0$.

Proof. Let $x \in M$ be a nonzero element. The assumption that $M \otimes_{R} R_{\infty}$ is almost zero implies that $\mathfrak{m} R_{\infty} \subset \operatorname{Ann}(x \otimes 1)$. Thus, the ideal $\operatorname{Ann}(x \otimes 1) \subset$ $R_{\infty}$ contains arbitrarily small $p$-powers. On the other hand, by the flatness of $R \rightarrow R_{\infty}$, we see that $\operatorname{Ann}(x \otimes 1)=\operatorname{Ann}(x) \otimes_{R} R_{\infty}$. As $I=\operatorname{Ann}(x)$ is an ideal in $R$ which is essentially of finite type over $V$, the smallest power $a$ of $p$ it contains is bounded above zero since $x \neq 0$. We formalize this by saying that the natural map $V \rightarrow R / I$ defined by 1 induces an injection

$$
V / p^{a} \hookrightarrow R / I
$$

for some rational number $a>0$. Base changing along the flat map $V \rightarrow \bar{V}$ gives an injection

$$
\bar{V} / p^{a} \hookrightarrow(R / I) \otimes_{V} \bar{V} \simeq(R / I) \otimes_{R} R_{\bar{V}} \simeq R_{\bar{V}} /\left(I \otimes_{R} R_{\bar{V}}\right),
$$

also induced by 1 . The assumption that $R_{\bar{V}} \rightarrow R_{\infty}$ is faithfully flat then shows that we have a composite injection induced by 1 as follows:

$$
\bar{V} / p^{a} \hookrightarrow R_{\bar{V}} /\left(I \otimes_{R} R_{\bar{V}}\right) \hookrightarrow R_{\infty} /\left(I \otimes_{R} R_{\infty}\right) .
$$

Since the ideal $I \otimes_{R} R_{\infty}=\operatorname{Ann}(x \otimes 1)$ contains arbitrarily small $p$-powers, so does its preimage under $\bar{V} \rightarrow R_{\infty}$. Hence, the map considered above is an injection only when $a=0$, which contradicts the assumption that $a>0$.

We are now in a position to prove Theorem 1.1.

Proof of Theorem 1.1. After replacing $V$ with its normalization in a suitable finite extension and replacing $R$ and $S$ by the corresponding base 
changes, we may assume that $R \otimes_{V} \bar{V}$ is a domain. Our goal, then, is to show that the exact sequence

$$
0 \rightarrow R \rightarrow S \rightarrow \mathcal{Q} \rightarrow 0
$$

is split, where $\mathcal{Q}$ is the cokernel. The obstruction to splitting is measured by an element $\mathrm{ob}(f) \in \operatorname{Ext}_{R}^{1}(\mathcal{Q}, R)$. We will show that this class almost vanishes after a suitable big extension, and then we apply Lemma 3.1.

The assumptions in the theorem imply that there exists an étale morphism $V\left[T_{1}, \ldots, T_{d}\right] \rightarrow R$ such that $R \rightarrow S$ is étale over $R\left[\left(1 / p T_{1}, \ldots, T_{d}\right)\right]$. Using this presentation, we define rings $R_{n}, S_{n}, R_{\infty}$, and $S_{\infty}$ as in Section 2.2. The picture over $R_{\infty}$ can be summarized as

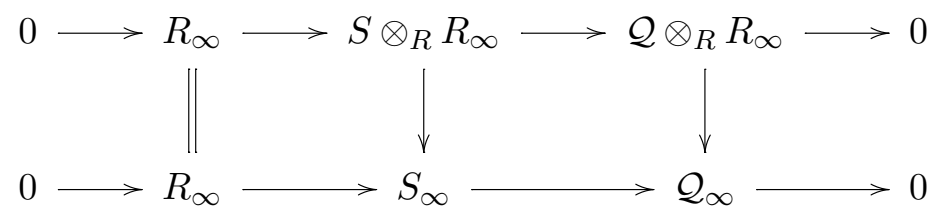

Here the first row is obtained by tensoring the exact sequence (1) with $R_{\infty}$, while $\mathcal{Q}_{\infty}$ is the cokernel of $R_{\infty} \rightarrow S_{\infty}$. Theorem 2.8 implies that the map $R_{\infty} \rightarrow S_{\infty}$ is an almost étale covering. By Lemma 2.7, the quotient $\mathcal{Q}_{\infty}$ is an almost projective $R_{\infty}$-module. Hence, the second exact sequence is almost zero when viewed as an element of $\operatorname{Ext}_{R_{\infty}}^{1}\left(\mathcal{Q}_{\infty}, R_{\infty}\right)$. The diagram above shows that the first exact sequence then defines an almost zero element of $\operatorname{Ext}_{R_{\infty}}^{1}\left(\mathcal{Q} \otimes_{R} R_{\infty}, R_{\infty}\right)$. On the other hand, the flatness of $R \rightarrow R_{\infty}$ shows that this element is simply ob $(f) \otimes 1$ under the natural isomorphism $\operatorname{Ext}_{R}^{1}(\mathcal{Q}, R) \otimes_{R} R_{\infty} \simeq \operatorname{Ext}_{R_{\infty}}^{1}\left(\mathcal{Q} \otimes_{R} R_{\infty}, R_{\infty}\right)$. By construction of $R_{\infty}$, the map $R_{\bar{V}} \rightarrow R_{\infty}$ is faithfully flat. Lemma 3.1 applied to the submodule of $\operatorname{Ext}_{R}^{1}(\mathcal{Q}, R)$ generated by ob $(f)$ shows that $\mathrm{ob}(f)=0$, so the theorem is proved.

REMARK 3.2. It is tempting to generalize Theorem 1.1 in two ways: (a) the base ring $R$ should be allowed to be semistable singularities, as Faltings's theorem from Section 2.2 works in that setting; and (b) the assumption on the ramification divisor should only be imposed on $f \otimes \mathbf{Q}$ following accepted philosophy in almost ring theory. While this article was being prepared for publication, both of these generalizations were carried out in [GR2, Section 10.7] using the ideas here. Unfortunately, the statement of the final result requires close to a thousand pages. 
REMARK 3.3. Scholze's recent work on perfectoid spaces [S], relying crucially on Huber's theory of adic spaces, as well as the theory of the cotangent complex, contains a sweeping generalization of Faltings's purity result used in the proof of Theorem 1.1. It seems very likely that his result (and the arguments of this article) can shed significant light on the direct summand conjecture in general; we plan to further investigate this idea in a future work.

Acknowledgments. I am very grateful to Mel Hochster for his comments on the manuscript, as well as numerous enlightening conversations about the direct summand conjecture over the years. Special thanks are also due Kiran Kedlaya, Paul Roberts, and Kazuma Shimomoto for useful conversations about this subject.

\section{REFERENCES}

[F1] G. Faltings, p-adic Hodge theory, J. Amer. Math. Soc. 1 (1988), 255-299. MR 0924705. DOI 10.2307/1990970.

[F2] , "Almost étale extensions" in Cohomologies p-adiques et applications arithmétiques, II, Astérisque 279, Soc. Math. France, Paris, 2002, 185-270. MR 1922831.

[GR1] O. Gabber and L. Ramero, Almost Ring Theory, Lecture Notes in Math. 1800, Springer, Berlin, 2003. MR 2004652.

[GR2] , Foundations of almost ring theory, preprint, arXiv:math/0409584v8 [math.AG].

[Gro] A. Grothendieck, Revêtements étales et groupe fondamental, Séminaire de Géométrie Algébrique du Bois-Marie (SGA 1), Doc. Math. (Paris) 3, Soc. Math. France, Paris, 2003. MR 2017446.

$[\mathrm{H}] \quad$ R. C. Heitmann, The direct summand conjecture in dimension three, Ann. of Math. (2) 156 (2002), 695-712. MR 1933722. DOI 10.2307/3597204.

[Ho1] M. Hochster, Contracted ideals from integral extensions of regular rings, Nagoya Math. J. 51 (1973), 25-43. MR 0349656.

[Ho2] , Canonical elements in local cohomology modules and the direct summand conjecture, J. Algebra 84 (1983), 503-553. MR 0723406. DOI 10.1016/ 0021-8693(83)90092-3.

[Ho3] , Homological conjectures, old and new, Illinois J. Math. 51 (2007), 151169. MR 2346192.

[O] M. C. Olsson, "On Faltings' method of almost étale extensions" in Algebraic Geometry (Seattle, 2005), Part 2, Proc. Sympos. Pure Math. 80 Part 2, Amer. Math. Soc., Providence, 2009, 811-936. MR 2483956.

[R] P. Roberts, Almost regular sequences and the monomial conjecture, Michigan Math. J. 57 (2008), 615-623. MR 2492472. DOI 10.1307/mmj/1220879428.

[S] P. Scholze, Perfectoid spaces, Publ. Math. Inst. Hautes Études Sci. 116 (2012), 245-313. MR 3090258. DOI 10.1007/s10240-012-0042-x. 
[T] J. T. Tate, "p-divisible groups" in Proceedings of a Conference on Local Fields (Driebergen, 1966), Springer, Berlin, 1967, 158-183. MR 0231827.

School of Mathematics

Institute for Advanced Study

Princeton, New Jersey 08540

USA

bhargav.bhatt@gmail.com 